\title{
Malignant mesothelioma eight years after a diagnosis of atypical mesothelial hyperplasia
}

\author{
James Scurry, Máire A Duggan
}

\begin{abstract}
The separation of mesothelial hyperplasia from early malignant mesothelioma remains one of the most difficult problems in histopathology. Inconclusive cases are termed "atypical mesothelial hyperplasia" and treated expectantly. A 49 year old male pipeline engineer was diagnosed as having atypical mesothelial hyperplasia in appendiceal serosa by the US-Canadian Mesothelioma Panel. Eight years later, he developed overtly malignant peritoneal and pleural mesothelioma. In hindsight, histological similarities between the diffuse malignant mesothelioma and the atypical mesothelial proliferation suggested malignancy from the outset. The most important of these features were the degree of mesothelial proliferation, micronodularity, architectural complexity, superficial invasion, uniform mild cytological atypia, and the absence of a clinical cause for a benign mesothelial proliferation. Ancillary investigations including immunohistochemistry were of no benefit in determining whether the atypical mesothelial hyperplasia was benign or malignant. Careful histological examination remains the mainstay of the diagnosis of early mesothelioma.

(f Clin Pathol 1999;52:535-537)
\end{abstract}

Keywords: atypical hyperplasia; mesothelium; malignant tumour; peritoneum

Department of Pathology, Mercy Hospital for Women, East Melbourne, Victoria, Australia J Scurry

Department of Pathology and

Laboratory Medicine, University of Calgary and Cytopathology

Division, Calgary

Laboratory Services, Calgary, Alberta,

Canada

M A Duggan

Correspondence to: Dr M A Duggan, Department of Pathology, Foothills Hospital, 1403-29th Street NW, Calgary, Alberta AB T2N 2T9, Canada email: maire.duggan@ crha-health.ab.ca

Accepted for publication 4 March 1999
One of the most difficult areas in histopathology is the separation of early well differentiated malignant mesothelioma from benign mesothelial hyperplasia. The term "atypical mesothelial hyperplasia" has been introduced for cases where the diagnosis is uncertain. In these cases, the proliferating mesothelium shows some but not all the histological or cytological features of well differentiated mesotheliomas. ${ }^{1}$ This term encompasses benign mesothelial hyperplasias, premalignant mesothelial proliferations, and early well differentiated maligis to observe patients with atypical mesothelial hyperplasia. Overt malignancy usually becomes manifest within months in cases where atypical mesothelial hyperplasia was in fact early mesothelioma. The justification for this approach is that surgery and adjuvant treatment in malignant mesothelioma have only a marginal effect on survival. To avoid morbidity to patients who in fact have reactive mesothelial hyperplasia, it is better to wait and see in doubtful cases. However, this approach is not ideal, as until premalignant and early diffuse nant mesotheliomas. The conventional wisdom malignant mesothelioma can be diagnosed reliably, it will be impossible to mount effective treatment trials for early disease. We present a case of atypical mesothelial hyperplasia found on the appendix in a 49 year old man who developed a diffuse malignant mesothelioma eight years later.

\section{Case report}

In 1989, a 49 year old male pipeline engineer, who had been working in fertiliser and gas processing plants on three continents since 1981, presented with right lower quadrant pain. A presumptive diagnosis of appendicitis was made. At laparotomy, the appendix and mesoappendix appeared thickened and were removed, but no other abnormality was noted. In 1994, he had two pulmonary emboli. At that stage, computed tomography of the abdomen showed thickened omentum and a small amount of free intraperitoneal fluid, but an ultrasound guided fine needle abdominal aspirate was negative for carcinoma. Extensive clinical, radiological, and biochemical investigation did not identify malignancy in any organ.

In late 1996, repeat computed tomography showed progressive thickening of the omentum, ascites, bilateral small pleural effusions, nodularity of the right greater pulmonary fissure, and mesenteric lymph node enlargement. In 1997, a right thoracocentesis for increasing pleural effusion revealed malignant cells on cytology. He underwent exploratory laparotomy. Ascites was confirmed, the peritoneal surface was diffusely thickened with small nodules of tumour, the omentum was infiltrated, and there were larger deposits of tumour in the right subphrenic space and on the spleen. Omental and mesenteric node biopsies were performed.

\section{Pathology}

External examination of the appendix (1989) showed pale thickening of the serosa and mesoappendix, but no tumour nodules were seen. Cut sections were normal. Histological examination showed no evidence of current or remote inflammation of the appendix. There was mild diffuse peritoneal fibrosis with scattered nests and small nodules of epithelioid cells up to $1.0 \mathrm{~mm}$ in diameter on and beneath the surface (fig 1). Most of the nests were solid, but there was a tendency for intercellular vacuoles and separation of the cells, so that rudimentary papillae were formed. The nests beneath the surface either communicated directly with fat, or were enveloped in a desmoplastic stroma. The cells had a strong 


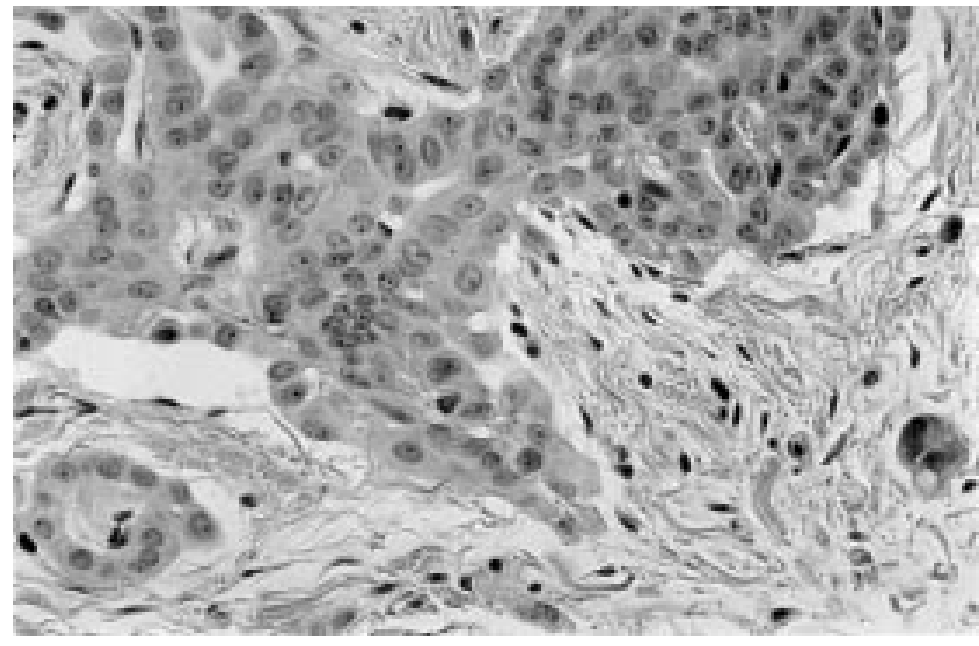

Figure 1 Periappendiceal mesothelial proliferation originally diagnosed as atypical mesothelial hyperplasia in a man who developed malignant mesothelioma eight years later. Note the worrying features of invasion, nodularity, complex architecture, and mild cytological atypia. (Haematoxylin and eosin, $\times 238$.)

resemblance to normal mesothelium, shown by a central location of their nuclei and dense eosinophilic cytoplasm, but with mildly atypical features of nuclear enlargement, increased nucleus to cytoplasm ratio, prominent and irregular eosinophilic nucleoli, and occasional mitotic figures. These cells formed a pure population, with no normal mesothelial cells present. There was no inflammatory infiltrate. A diagnosis of atypical mesothelial hyperplasia was made by the United States-Canadian Mesothelioma Panel, Ottawa, Ontario, Canada.

Cytological examination of the pleural fluid (1997) showed tightly aggregated balls of atypical monomorphic epithelioid cells, analogous to those seen in the atypical mesothelial hyperplasia.

The omental biopsy (1997) consisted of two fragments of fat, 30 and $20 \mathrm{~mm}$ in maximum dimension, diffusely infiltrated by solid pale tumour. Histologically, the tumour showed a

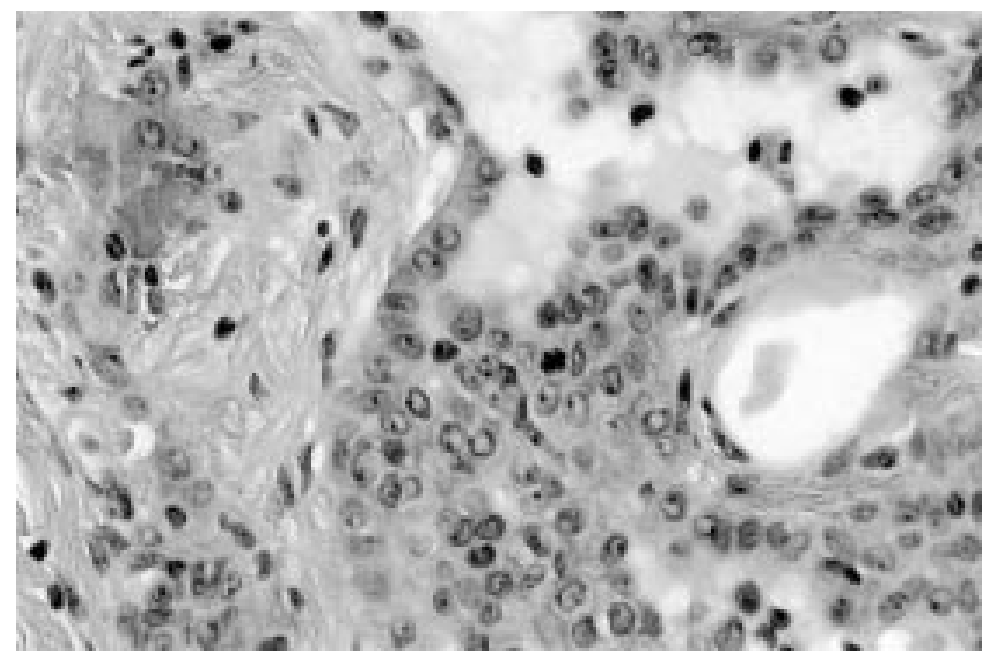

Figure 2 Omentum showing diffuse malignant mesothelioma. The similarities with the earlier periappendiceal biopsy in fig 1 make it difficult to decide which figure is of atypical mesothelial hyperplasia and which is of diffuse malignant mesothelioma; they suggest in hindsight that the earlier biopsy was probably already malignant mesothelioma. There are, however, subtle differences in this later biopsy of slightly more complex architecture, cytological atypia, and mitosis which make it more readily recognisable as malignant. (Haematoxylin and eosin, $\times 238$.) tendency to have a papillary surface and a solid infiltrating region. The infiltrating portion was associated with a desmoplastic reaction and a lymphocytic infiltrate. Occasional psammoma bodies were present. The tumour cells were similar to those seen in the serosa of the appendix (fig 2), but showed a slightly higher degree of atypia. The mesenteric lymph node showed hyperplastic changes.

Mesothelial differentiation in the appendiceal and omental cellular proliferations was confirmed by the findings of alcian blue positive, hyaluronidase sensitive extracellular mucin, cytoplasmic border epithelial membrane antigen and intracytoplasmic cytokeratin immunostaining, and typical electron microscopic features including numerous long microvilli and extracellular spaces. Immunohistochemistry for p53 and Ki67 in both specimens was completely negative. Benign reactive mesothelium from a hernial sac of another patient used as a control showed occasional variably strong nuclear p53 reactivity in 5\% of cells and Ki67 reactivity in $10 \%$ of cells. Flow cytometry performed on the omental tumour showed a purely normal DNA content, with $100 \%$ of the cells diploid and the DNA index 1.00, with 6\% of cells in G2 phase and $2.8 \%$ in S phase.

A final diagnosis of diffuse malignant mesothelioma, epithelioid type, involving the peritoneum and pleura was made. An asbestos fibre count on tumour tissue was not performed.

\section{Discussion}

The confinement to serous cavities, histological features of a solid and tubopapillary epithelioid tumour, cytological features of cells with eosinophilic cytoplasm and central nuclei, hyaluronidase sensitive extracellular mucin, cytoplasmic keratin, cytoplasmic border epithelial membrane antigen, and electron microscopic features of the brush-like long microvilli all led to a diagnosis of diffuse epithelioid malignant mesothelioma in 1997. This was supported by the absence of any clinical or laboratory features to suggest an extraserosal primary site of malignancy.

The histological similarities between the tumour and the earlier serosal proliferation on the appendix from 1989 suggest that the tumour developed from this serosal proliferation. Originally, the serosal changes were diagnosed as "atypical mesothelial hyperplasia" by the US-Canadian Mesothelioma Panel. Although all panel members favoured a benign prognosis, the chairman (Dr E McCaughey) cautioned that he had not previously seen such a degree of proliferation in a benign case. In hindsight, the similarity with the diffuse malignant mesothelioma, degree of proliferation, stromal invasion, architectural complexity, uniformly mild cytological atypia, and absence of any potential cause of mesothelial hyperplasia all suggest early malignancy.

Sixty five per cent of malignant mesotheliomas are diploid as in this case, so if this investigation had been performed on the atypical mesothelial proliferation, it would not have been helpful. ${ }^{2}$ p53 Immunostaining has been 
Table 1 Summary of differentiating features between mesothelial hyperplasia and diffuse malignant mesothelioma (from references 1-10)

\begin{tabular}{|c|c|c|}
\hline & Benign & Malignant \\
\hline History & Younger; history of chronic infection; therapeutic radiation & Older; asbestos exposure \\
\hline Gross & $\begin{array}{l}\text { Localised to outpouchings of the peritoneal cavity such as } \\
\text { herniae; smooth }\end{array}$ & Diffusely involving serosa; nodular \\
\hline \multirow[t]{2}{*}{ Histology } & $\begin{array}{l}\text { Lower degree of proliferation; clumps of cells held together by } \\
\text { fibrin, with inflammatory cells; limited to serosal surfaces, } \\
\text { rarely superficially invades; no necrosis }\end{array}$ & $\begin{array}{l}\text { Higher degree of proliferation; } \\
\text { tubopapillary structures; invasive; necrosis }\end{array}$ \\
\hline & Uniformity of cells; mitoses rare & Cytological atypia; mitoses common \\
\hline \multirow[t]{3}{*}{ Cytology } & Moderately hypercellular & Markedly hypercellular \\
\hline & Smaller less complex groups, flat sheets & $\begin{array}{l}\text { Large complex groups, irregularly three } \\
\text { dimensional, with papillae and cell in cell } \\
\text { formation }\end{array}$ \\
\hline & $\begin{array}{l}\text { Large but not macronucleoli; monomorphism; reactive } \\
\text { features }\end{array}$ & $\begin{array}{l}\text { Macronucleoli; pleomorphism; malignant } \\
\text { features }\end{array}$ \\
\hline Immunoperoxidase & p53 negative & p53 positive in $44 \%$ \\
\hline Ploidy & Diploid & Aneuploid in $35 \%$ \\
\hline Cytogenetics & Normal & Usually abnormal, but no specific changes \\
\hline
\end{tabular}

used to distinguish benign mesothelial proliferations from malignant mesothelioma, but was only present in 63 of 143 mesotheliomas (44\%) when four studies are combined..$^{3-6}$ Our results of negative atypical mesothelial hyperplasia and mesothelioma, but focal positivity in $5 \%$ of reactive mesothelial cells in a negative control urge caution in interpreting p53 immunostaining in mesothelial proliferations. Wild-type p53 may be stained by the commonly used antibodies. ${ }^{7}$

A summary of the diagnostic features distinguishing mesothelial hyperplasia from early mesothelioma is given in table 1 . Careful histological examination remains the key to diagnosis.

Eighty to ninety per cent of mesotheliomas in the United Kingdom appear to be related to previous asbestos exposure. ${ }^{8}$ Our patient had a 16 year history of potential occupational asbestos exposure by the time a definitive diagnosis of malignant mesothelioma was made in 1997. Ninety nine per cent of 1690 patients with mesotheliomas had a latent period of at least 15 years, and in $96 \%$ it was 20 years. ${ }^{9}$ The original mesothelial proliferation occurred after only eight years of potential exposure to asbestos, and supports the concept that the atypical mesothelial hyperplasia was malignant at the time of the appendectomy.

1 McCaughey WTE, Al-Jabi M. Differentiation of serosal hyperplasia and neoplasia in biopsies. Pathol Annu 1986;1:271-93.

2 Burmer GC, Rabinovitch PS, Kulander BG, et al. Flow cytometric analysis of malignant pleural mesotheliomas. Hum Pathol 1989;20:777-83.

3 Ramael M, Lemmens G, Eerdekens C, et al. Immunoreactivity for p 53 protein in malignant mesothelioma and nonneoplastic mesothelium. F Pathol 1992;168:371-5.

4 Mayall FG, Goddard H, Gibbs AR. p53 Immunostaining in the distinction between benign and malignant mesothelial proliferations using formalin-fixed paraffin sections. F Pathol 1992;168:377-81.

5 Kafiri G, Thomas GM, Shepherd NA, et al. p53 Expression is common in malignant mesothelioma. Histopathology 1992;21:331-4

6 Cagle PT, Brown RW, Lebovitz RM. p53 Immunostaining in the differentiation of reactive processes from malignancy in pleural biopsy specimens. Hum Pathol 1994;25:443-8.

7 Attanoos RL, Gibbs AR. Pathology of malignant mesothelioma. Histopathology 1997;30:403-18.

8 Lanphear BP, Buncher CR. Latent period of malignant mesothelioma of occupational origin. F Occup Med 1992;7: $718-22$

9 Enzinger FM, Weiss SW. Mesothelioma. In: Stamathus G, ed. Soft tissue tumors, 3rd ed. St. Louis: CV Mosby, 1995:797-819.

10 DeMay RM. Fluids. In: Philips R, ed. The art and science of ety of Clinical Pathologists, 1995:257-325. 